INPLASY

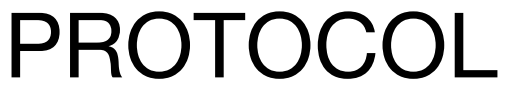

To cite: Tan et al.

Reconstruction of anterior cruciate ligament with hamstring tendon versus allogeneic tendon: A metaanalysis. Inplasy protocol

202150111. doi:

10.37766/inplasy2021.5.0111

Received: 31 May 2021

Published: 31 May 2021

Corresponding author:

Chujie Chen

23612212@qq.com

Author Affiliation:

Guangzhou University of

Chnise Medicine.

Support: Special funds.

Review Stage at time of this submission: Risk of bias assessment.

Conflicts of interest:

None declared.

\section{Reconstruction of anterior cruciate ligament with hamstring tendon versus allogeneic tendon: A meta-analysis}

Tan, $\mathrm{H}^{1}$; Chen, C2; L, B3; W, X4.

Review question / Objective: Compare the effect of anterior cruciate ligament reconstruction with autologous tendon and allogeneic tendon.

Rationale: Autogenous tendon and allogeneic tendon have their own advantages and disadvantages. How to choose the appropriate tendon according to the specific situation is still not have a final conclusion. In this study, 8 databases at home and abroad were searched for relevant retrospective studies and randomized controlled studies to compare their differences in postoperative subjective functional recovery, return to exercise and knee arthritis and so on.

INPLASY registration number: This protocol was registered with the International Platform of Registered Systematic Review and Meta-Analysis Protocols (INPLASY) on 31 May 2021 and was last updated on 31 May 2021 (registration number INPLASY202150111).

Rationale: Autogenous tendon and allogeneic tendon have their own

advantages and disadvantages. How to choose the appropriate tendon according to the specific situation is still not have a final conclusion. In this study, 8 databases at home and abroad were searched for relevant retrospective studies and randomized controlled studies to compare their differences in postoperative 
subjective functional recovery, return to exercise and knee arthritis and so on.

Condition being studied: Reconstruction of anterior cruciate ligament with hamstring tendon versus allogeneic tendon. Literature: the library of Guangzhou University of traditional Chinese medicine has a variety of medical books and journals, and the electronic reading room can retrieve and consult the latest literature at home and abroad, which can meet the needs of this study; For two different kinds of tendon reconstruction of anterior cruciate ligament, several databases have more studie, which can meet the needs of this project; Associate Professor Chujie Chen, the instructor of this project, has rich scientific research experience and can guide the progress of this project complete smoothly.

\section{METHODS}

Search strategy: Terms:Anterior Cruciate Ligament Injuries, Autografts, Allografts, Hamstring Tendons .Electronic databases: Pubmed, Embase, Cochrane Library, Web of science, China National Knowledge Infrastructue, China Science and Technology Journal Database, WanFang Data and China Biology Medicine disc.

Participant or population: The number of cases in the relevant retrospective literature and randomized controlled literature were retrieved from the database.

Intervention: Anterior cruciate ligament reconstruction with hamstring autograft.

Comparator: Anterior cruciate ligament reconstruction with allogeneic tendon.

Study designs to be included: The types of studies collected were based on randomized controlled and retrospective control tests. PubMed, EMBASE, Cochrane Library and other databases were searched to select articles according to the inclusion and exclusion criteria. According to Jadad scale and NOS scale, the qualified literatures were evaluated to exclude highrisk literatures. the basic information of literatures was extracted, and the IKDC, Tegner score, number of Kellgren Lawrence, number of axial displacement test grade I, number of axial displacement test grade II and number of axial displacement test grade III were recorded respectively. Use Review Manager 5.3 to compare the differences between the two.

Eligibility criteria: (1) The types of studies collected were based on randomized controlled and retrospective control tests. (2) The subjects of the study were patients who needed reconstruction of ACL injury(3) The experimental group was reconstruction of the hamstring tendon and the control measures were allogeneic tendon reconstruction(4) The outcome measures: 1) IKDC score was evaluated at the last follow-up, and the knee function was evaluated; 2) KL positive (grade II, grade III, IV) was used to evaluate the postoperative knee arthritis; 3) The number of ROM positive (grade III and IV) was evaluated; 4) Tegner score was used to evaluate the postoperative return to exercise; 5) The number of people in the axial shift test was grade I, and the ligament relaxation was evaluated; 6) The total number of grade II and III was evaluated.

Information sources: PubMed, EMBASE, Cochrane Library and other databases were searched to select articles according to the inclusion and exclusion criteria. The related retrospective literature and randomized controlled literature were retrieved from the database.

Main outcome(s): IKDC score was evaluated at the last follow-up, and the knee function was evaluated; KL positive (grade II, grade III, IV) was used to evaluate the postoperative knee arthritis; The number of ROM positive (grade III and IV) was evaluated; Tegner score was used to evaluate the postoperative return to exercise; 5) The number of people in the axial shift test was grade $I$, and the ligament relaxation was evaluated; The total number of grade II and III was evaluated. 
Data management: Noteexpress software management related literature.

Quality assessment / Risk of bias analysis: Jadad scale and NOS scale.

Strategy of data synthesis: Reveman manager 5.3 was used to analyze the fixed effect model of the selected literature. If 12 $<50 \%, P>0.1$ and the sensitivity was low, the relationship between the data and the invalid line was compared. If $12>50 \%, P<$ 0.1 , subgroup study was used to explore the reasons and differences.

Subgroup analysis: Included IKDC score at the last follow-up; The number of $K L$ positive (grade II, III, IV) was higher; The number of ROM positive patients (grade III and IV) was higher than that of the control group; Tegner score, axial displacement test grade I number of people; Total number of class II and III.

Sensitivity analysis: Randomly delete a literature in the study to see the change of numerical value.

Language: English.

Country(ies) involved: China.

Keywords: anterior cruciate ligament, autologous, hamstring tendon, allogeneic tendon.

Contributions of each author:

Author 1 - Haoyin Tan.

Email: 969486000@qq.com

Author 2 - Chujie Chen.

Email: 23612212@qq.com

Author 3 - Bin, L.

Author 4 - Xin, W.

Author 1 draft literatures, select literatures and meta analysis; Author 2 and author 3 screened literatures and evaluated their quality; corresponding authors provide technical guidance and meta analysis. 